\title{
X-rays from RU Lupi: accretion and winds in classical T Tauri stars
}

\author{
J. Robrade and J. H. M. M. Schmitt
}

Universität Hamburg, Hamburger Sternwarte, Gojenbergsweg 112, 21029 Hamburg, Germany

e-mail: jrobrade@hs . uni-hamburg.de

Received 13 April 2007 / Accepted 18 June 2007

\section{ABSTRACT}

\begin{abstract}
Context. Low-mass stars are known to exhibit strong X-ray emission during their early evolutionary stages. This also applies to classical T Tauri stars (CTTS), whose X-ray emission differs from that of main-sequence stars in a number of aspects.

Aims. We study the specific case of RU Lup, a well known accreting and wind-driving CTTS. In comparison with other bright CTTS we study possible signatures of accretion and winds in their X-ray emission.

Methods. Using three XMM-Newton observations of RU Lup, we investigate its X-ray properties and their generating mechanisms. High-resolution X-ray spectra of RU Lup and other CTTS are compared to main-sequence stars. We examine the presence of a cool plasma excess and enhanced plasma density in relation to X-rays from accretion shocks and investigate anomalous strong X-ray absorption and its connection to winds or circumstellar material.

Results. We find three distinguishable levels of activity among the observations of RU Lup. While no large flares are present, this variability is clearly of magnetic origin due to the corresponding plasma temperatures of around $30 \mathrm{MK}$; in contrast the cool plasma component at 2-3 MK is quite stable over a month, resulting in a drop of average plasma temperature from $35 \mathrm{MK}$ down to $10 \mathrm{MK}$. Density analysis with the O VII triplet indicates high densities in the cool plasma, suggesting accretion shocks to be a significant contributor to the soft X-ray emission. No strong overall metal depletion is observed, with Ne being more abundant than Fe, that is at solar value, and especially $\mathrm{O}$. Excess emission at $6.4 \mathrm{keV}$ during the more active phase suggest the presence of iron fluorescence. Additionally RU Lup exhibits an extraordinary strong X-ray absorption, incompatible with estimates obtained at optical and UV wavelengths. Comparing spectra from a sample of main-sequence stars with those of accreting stars we find an excess of cool plasma as evidenced by lower O VIII/O VII line ratios in all accreting stars. High density plasma appears to be only present in low-mass CTTS, while accreting stars with intermediate masses $\left(\gtrsim 2 M_{\odot}\right)$ have lower densities.

Conclusions. In all investigated CTTS the characteristics of the cooler X-ray emitting plasma are influenced by the accretion process. We suspect different accretion rates and amounts of funnelling, possibly linked to stellar mass and radius, to be mainly responsible for the different properties of their cool plasma component. The exceptional X-ray absorption in RU Lup and other CTTS is probably related to the accretion flows and an optically transparent wind emanating from the star or the disk.
\end{abstract}

Key words. stars: individual: RU Lupi - stars: pre-main sequence - stars: activity - stars: coronae - X-rays: stars

\section{Introduction}

T Tauri stars as a class, are very young low-mass stars. The members of the subclass of so-called classical T Tauri stars (CTTS) are still accreting matter from a surrounding circumstellar disk. CTTS are thought to evolve first into weak-line T Tauri stars (WTTS), where they become virtually disk-less and no longer show signs of significant accretion, and eventually into solarlike star on the main sequence. Ongoing accretion in a CTTS is evidenced by an emission line spectrum and specifically, a large $\mathrm{H} \alpha$ equivalent width $(E W>10 \AA)$. Further, the strong observed infrared excess indicates the presence of a disk. In contrast, WTTS have much weaker $\mathrm{H} \alpha$ emission and little or no IR-excess. The increasing dominance of the underlying continuum emission over the optical stellar absorption line spectrum gives rise to another sub-classification into moderate, veiled and extreme T Tauri stars. However, these strict subdivisions are somewhat arbitrary and blurred since for example $\mathrm{H} \alpha$ emission is highly variable and differs also within the CTTS class by more than an order of magnitude. A detailed account of the pre-main sequence stellar evolution of low-mass stars is given by Feigelson \& Montmerle (1999).

In the commonly accepted magnetospheric accretion model for CTTS (Calvet \& Gullbring 1998) material is assumed to be accreted from the stellar disk onto the star at almost free-fall velocity along magnetic field lines which disrupt the accretion disk in the vicinity of the corotation radius. Upon impact, a strong shock is formed near the stellar surface and the funnelling of the accreted matter by the magnetic field leads to "accretion spots" with filling factors at the percent level with respect to the stellar surface; therefore only a small fraction of the stellar surface is covered by the accretion spots. This accretion shock plasma is expected to reach temperatures of up to a few MK and thus to lose a large fraction of its energy at UV and soft X-ray wavelengths. The accreted plasma does not necessarily have to be at high density. However, if one wants to achieve the inferred small filling factors at the percent level and the otherwise determined mass accretion rates of $10^{-8}-10^{-7} M_{\odot} \mathrm{yr}^{-1}$, the infalling gas must then be at rather high density $\left(n>10^{11} \mathrm{~cm}^{-3}\right)$. The accretion process is accompanied by outflows from the star and possibly also from the disk, which probably also play an important role in the transport of angular momentum. Different mass accretion rates and filling factors, different stellar properties such as mass, rotation and activity and different viewing angles, naturally lead to the observed variety of the different CTTS phenomena.

X-ray emission from T Tauri stars has already been detected with the Einstein and ROSAT observatories, and both types are found to be copious and variable X-ray emitters. The origin of 
the observed X-ray emission is currently the subject of some debate. Since all main-sequence "cool stars", i.e. stars with outer convection zones, are surrounded by X-ray emitting coronae (Schmitt \& Liefke 2004), some kind of magnetic activity is also expected for their pre-main sequence counterparts with their deep outer convection zones. Indeed, large sample studies such as COUP (Chandra Orion Ultradeep Project) showed that most of their observed X-ray emission is of coronal origin (Preibisch et al. 2005). Further, magnetic fields, connecting star and disk, have been invoked to explain the huge flares observed in CTTS (Favata et al. 2005a).

On the other hand, the presence of accretion streams and outflows opens up additional possibilities for the generation of X-rays. Collimated winds have been observed as X-ray jets in Herbig-Haro objects (Pravdo et al. 2001; Favata et al. 2006) and in CTTS such as DG Tau (Güdel et al. 2005). Accretion shocks are expected to generate plasma at a significantly higher density than stellar coronal plasma and their soft X-ray emission can be traced by density sensitive line ratios, e.g. between forbidden and intercombination lines in the He-like triplets of O VII and Ne IX. Anomalously low ratios of these lines have been observed for several CTTS, e.g. TW Hya (Kastner et al. 2002; Stelzer \& Schmitt 2004), BP Tau (Schmitt et al. 2005), CR Cha (Robrade \& Schmitt 2006), V4046 Sgr (Günther et al. 2006) and MP Mus (Argiroffi et al. 2007), and the inferred high plasma densities suggest the presence of X-ray emission from shocks as postulated in the magnetospheric accretion scenario.

However, not all accreting stars seem to show high density plasma. The prototypical, but not necessarily typical T Tauri star, $\mathrm{T}$ Tau itself, is an example where density diagnostics of cool plasma as seen in O VII indicate a lower density which is more comparable to active main sequence stars (Güdel et al. 2007). Further, accretion is only capable of producing plasma with temperatures of up to a few MK (e.g. Günther et al. 2007), and therefore contributes nearly exclusively to the low energy X-ray emission. However, in all CTTS - even in the accretion dominated, "soft" TW Hya - high temperature plasma is observed, which together with the strong flaring requires magnetic activity to also be present in these objects. A comparative study of several bright CTTS indicated the presence of X-rays from both accretion and magnetic activity, but with different respective contributions in the individual objects (Robrade \& Schmitt 2006). Altogether, magnetic activity is the dominant source of the X-ray emission in most CTTS at least at higher energies, nonetheless accretion can significantly contribute to the soft X-ray emission.

RU Lup is a late K-type CTTS with a high accretion rate and an extreme optical veiling. It has been extensively studied especially at optical and UV wavelengths and a review of its basic stellar properties is given by Lamzin et al. (1996) and Stempels \& Piskunov (2002). Lamzin et al. (1996) present a magnetospheric accretion model for RU Lup based on multi-frequency monitoring and high-resolution optical data. While there was some debate about the distance of RU Lup (140-250 pc), recent estimates indicate a value of $\sim 140 \mathrm{pc}$ (Hughes et al. 1993), which we adopt for this paper. Already Lamzin et al. (1996) pointed out that their derived reddening sets an upper limit to RU Lup's distance of $\sim 150 \mathrm{pc}$, when attributed only to interstellar material, also indicating very little circumstellar absorption. RU Lup is highly variable and shows strong accretion signatures with a large $\mathrm{H} \alpha E W$ of $140 \AA$ up to $216 \AA$ (Appenzeller et al. 1983). Virtually no photospheric absorption lines are detected in its spectrum due to substantial optical veiling, on the contrary, the optical spectrum shows many emission lines. The line profiles are also strongly variable and asymmetric, suggesting
Table 1. Observing log of the XMM-Newton RU Lup exposures.

\begin{tabular}{rrrr}
\hline \multicolumn{1}{l}{ Observation } & MOS (filt.) & OM \\
Date & Time & Dur.(ks) & Exp.(no.) \\
\hline August 08 2005 & $04: 19-12: 54$ & $30(28)$ & 6 \\
August 17 2005 & $09: 17-17: 02$ & $27(15)$ & 6 \\
Sept. 06/07 2005 & $18: 30-02: 16$ & $27(22)$ & 6 \\
\hline
\end{tabular}

both infalls and outflows with velocities up to $300-400 \mathrm{~km} \mathrm{~s}^{-1}$. Specifically, Lamzin (2000) attributes the origin of the observed lines profiles in the HST UV spectra to accretion shocks and stellar winds.

From optical high-resolution spectra, Stempels \& Piskunov (2002) determined mass and radius of RU Lup to $M \sim 0.8 M_{\odot}$ and $R \sim 1.7 R_{\odot}$. They further derived a projected rotational velocity of $v \sin (i)=9 \mathrm{~km} \mathrm{~s}^{-1}$ and detected variability on short time scales of $1 \mathrm{~h}$. Together with various proposed periods $(0.8-$ $3.7 \mathrm{~d})$ this suggests an inclination between $3^{\circ}$ and $16^{\circ}$, i.e. a system that is viewed nearly pole-on with rather high intrinsic rotation. Finally, they derived an interstellar absorption column towards RU Lup with a corresponding $N(\mathrm{H})=6.3 \times 10^{19} \mathrm{~cm}^{-2}$. Combining literature data with results derived from FUV data obtained with FUSE and HST/STIS, Herczeg et al. (2005) estimated RU Lup to be an $0.6-0.7 M_{\odot}$ star with an age of 2-3 Myr and a mass accretion rate of $\sim 5 \times 10^{-8} M_{\odot}$. Their derived hydrogen column density of $\log N(\mathrm{H})=20.1 \pm 0.2 \mathrm{~cm}^{-2}$, corresponding to an extinction of $A_{\mathrm{V}} \sim 0.07$, is again quite low. Combining and summarising these findings we conclude that RU Lup is probably an optically rather mildly absorbed system viewed almost pole-on.

While the Einstein IPC failed to detect X-ray emission from RU Lup with an upper flux limit of $1.2 \times 10^{-13} \mathrm{erg} \mathrm{cm}^{-2} \mathrm{~s}^{-1}$ in the $0.5-4.0 \mathrm{keV}$ band (Gahm 1980), X-ray emission from RU Lup was detected in 1993 in a pointed $15.7 \mathrm{ks}$ ROSAT PSPC $(0.1-2.4 \mathrm{keV})$ observation at a rate of $9 \times 10^{-3} \mathrm{cts} / \mathrm{s}$ (2RXP catalogue). Using the energy conversion factors given by Neuhäuser et al. (1995), this corresponds to an X-ray flux of $\sim 1 \times 10^{-13} \mathrm{erg} \mathrm{cm}^{-2} \mathrm{~s}^{-1}$ and an X-ray luminosity of $\log L_{\mathrm{X}}=$ $29.4 \mathrm{erg} \mathrm{s}^{-1}$ for a distance of $140 \mathrm{pc}$.

In this paper we present three new XMM-Newton observations of RU Lup with a total exposure time of $84 \mathrm{ks}$, performed over the course of a month in 2005. Our paper is structured as follows. In Sect. 2 the X-ray observations and the data analysis are described, in Sect. 3 we present our results derived from the XMM-Newton observations of RU Lup subdivided into different physical topics. Finally, in Sect. 4 we compare RU Lup to results from X-ray diagnostics of several CTTS and specifically discuss accretion and wind scenarios in the context of their X-ray emission in general, which is followed by our conclusions in Sect. 5.

\section{Observations and data analysis}

RU Lup was observed by XMM-Newton three times in the course of a month in August/September 2005 for roughly $30 \mathrm{ks}$ each; the detailed observation times are listed in Table 1. Data were taken with all X-ray detectors, the EPIC (European Photon Imaging Camera), consisting of the MOS and PN detectors and the RGS (Reflection Grating Spectrometer) as well as the optical monitor (OM). The EPIC instruments were operated in full frame mode with the medium filter, while the OM was operated with the UVW1 filter, which is sensitive in the waveband between $2000-4000 \AA$ with an effective wavelength of $2910 \AA$. The OM exposures typically lasted around $4 \mathrm{ks}$ and allowed an 
accurate determination of RU Lup's UV brightness. During each X-ray observation, six exposures of the central field containing RU Lup were taken.

All data analysis was carried out with the XMM-Newton Science Analysis System (SAS) version 7.0 (Loiseau et al. 2006). Standard selection criteria were applied to the data, and periods with enhanced background due to proton flares were discarded from spectral analysis utilising the high energy event rates of the respective detectors. All X-ray light curves are background subtracted, the background was taken from nearby source-free regions. Spectral analysis of the EPIC data was carried out with XSPEC version 11.3 (Arnaud 1996) and is performed in the energy range $0.2-10.0 \mathrm{keV}$. The RGS data of the individual observations is of very low SNR, we therefore merged the data from all three observations using the tool rgscombine. We note that the X-ray source RX J1556.4-3749 of unknown type lies in the read-out direction of the RGS, the offset in the dispersed spectra is around -0.5 $\AA$. Therefore RX J1556.4-3749 could in principle, contaminate the RGS spectra of RU Lup, but being substantially absorbed, as deduced from its EPIC spectra, its contribution to the spectral lines and especially the O VII triplet discussed here is negligible. To reduce background and detector noise effects we extracted RGS spectra from the standard source region at phases of very low background (95\% PSF, background rate $<0.25 \mathrm{cts} / \mathrm{s}$ ) as well as from the line core at low background ( $66 \%$ PSF, background rate $<1 \mathrm{cts} / \mathrm{s}$ ) and crosschecked the derived results. For line fitting purposes we use the CORA program (Ness \& Wichmann 2002), using identical line width and assuming Lorentzian line shapes. Emitted line fluxes are corrected for absorption by using the ismtau-tool provided in the PINTofALE software (Kashyap \& Drake 2000).

The data of the EPIC detectors are analysed simultaneously for each observation but were not co-added. They are modelled with a multi-temperature model assuming the emission spectrum of a collisionally-ionized, optically-thin gas as calculated with the APEC code (Smith et al. 2001). We find that a threetemperature model adequately describes the data. Absorption in the circumstellar environment and in the interstellar medium is significant and is applied in our modelling as a free parameter. Abundances are modelled relative to solar photospheric values as given by Grevesse \& Sauval (1998). The low FIP (First Ionisation Potential) elements $\mathrm{Al}, \mathrm{Ca}, \mathrm{Ni}$ are tied to the $\mathrm{Fe}$ abundance; for other elements with no significant features in the measured X-ray spectra, solar abundances are used. Since absorption, the coolest plasma temperature and abundances do not significantly differ between the observations, they are modelled with variable, but tied values to ensure a consistent analysis of the data. We then derived the temperatures and volume emission measures ( $\left.E M=\int n_{\mathrm{e}} n_{\mathrm{H}} \mathrm{d} V\right)$ of the individual plasma components for all three observations and calculated corresponding $\mathrm{X}$-ray luminosities from the resulting best fit models. Some of the fit parameters are mutually dependent. This interdependence mainly affects the strength of absorption and emission measure of the cooler plasma at a few MK, but also components of the emission measure distribution (EMD) and abundances of elements with emission lines in the respective temperature range. Consequently, models with different absolute values of these parameters but only marginal differences in its statistical quality may be applied to describe the data.

Our fit procedure is based on $\chi^{2}$ minimization, therefore spectra were rebinned to satisfy the statistical demand of a minimum value of 15 counts per spectral bin. All errors derived in spectral fitting are statistical errors given by their $90 \%$

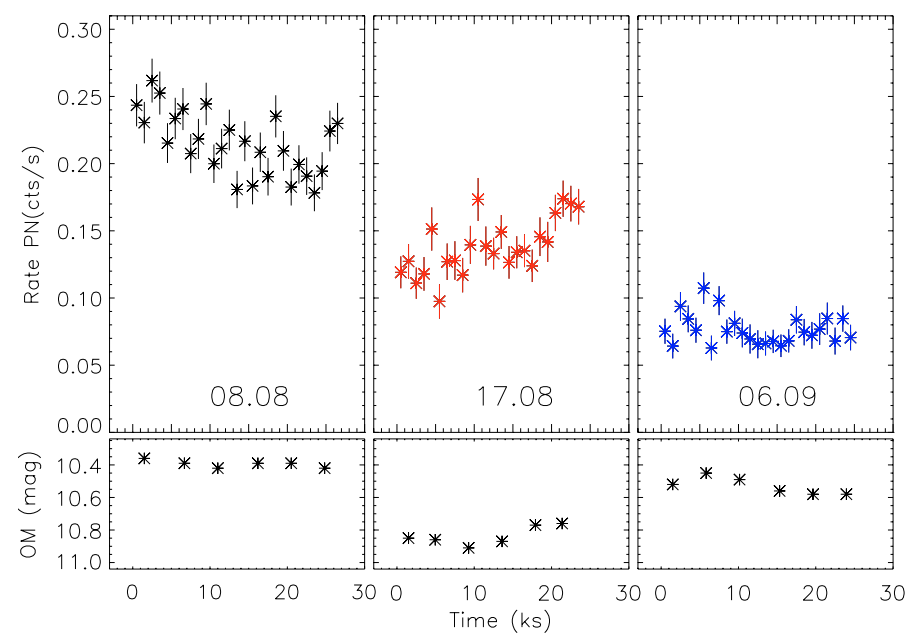

Fig. 1. Light curves of RU Lup determined with the PN instrument (1 ks binning) in the energy band $0.2-5.0 \mathrm{keV}$ (upper panels) and OM UVW1 magnitude per exposure (lower panels).

confidence range and were calculated separately for abundances and temperatures by allowing variations of normalisations and respective model parameters. Note that additional uncertainties may arise from errors in the atomic data and instrumental calibration which are not explicitly accounted for.

\section{Results}

\subsection{X-ray light curves and variability}

The XMM-Newton X-ray light curves of RU Lup are shown in the upper panel of Fig. 1. We observe a steady decline in X-ray brightness by a factor of three over a month. Note that because of the large time gaps between the observations ( 9 and 20 days respectively) this decline does not need to be monotonic. Indeed, already in the first observation, $L_{X}$ declined by roughly $40 \%$ within $25 \mathrm{ks}$ but started to rise again towards the end of the observation. During the second observation the count rate increased by roughly $50 \%$ and short-term X-ray variability is quite common in all observations. We can rule out the presence of strong flaring within the individual observations, however the August 08 light curve may be interpreted as a part of the decline phase of a larger flare. At any rate, the observed overall decline during the XMM-Newton observations is very likely accidental due to sparse temporal sampling.

The OM UVW1 flux observed from RU Lup is plotted in the lower panel of Fig. 1. Note that derived magnitude errors are in the range of $0.01 \mathrm{mag}$ and below the size of the shown symbols. We find variations in UVW1 brightness of up to 0.5 mag between the three observations, however these variations do not correlate with the observed X-ray brightness and have a far lower amplitude compared to the variations seen in the X-rays. Additional UVW1 variability is observed within each observation of the order $0.1-0.15 \mathrm{mag}$. Some of these variations appear to be correlated with the X-ray flux, possibly in the second and especially during the third and X-ray faintest observation. Unfortunately a time-resolved X-ray spectral analysis within each observation suffers from the poor signal and a correlation for six exposures might be a chance effect. Nonetheless, the fact that the correlation is most strongly present during the low-activity phases, where the soft X-ray component becomes more important, supports the view that at least some of the X-ray emission might be caused by the same physical process as the observed UV 


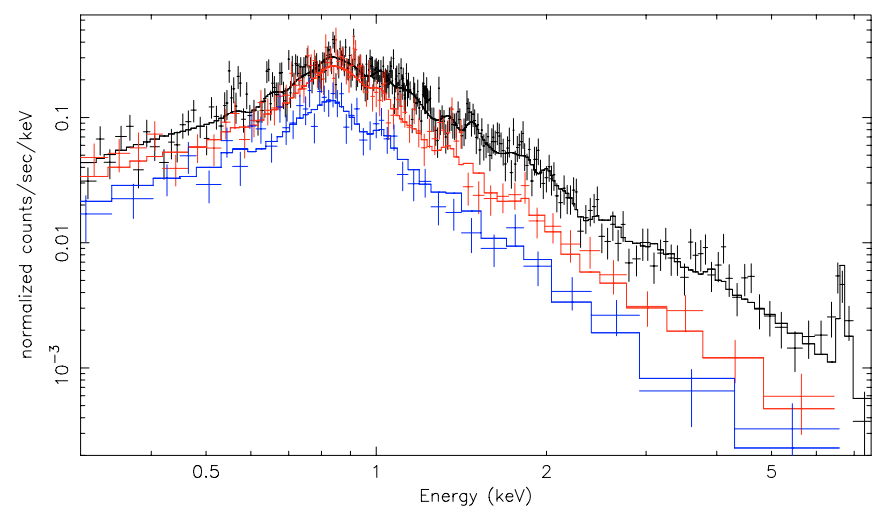

Fig. 2. X-ray spectra (PN) of RU Lup (crosses) and spectral fit (histogram) for the three observations; top down: 08/08 (black), 17/08 (red), 06/09 (blue).

Table 2. Spectral fit results for RU Lup, units are $N_{\mathrm{H}}$ in $10^{21} \mathrm{~cm}^{-2}, k T$ in $\mathrm{keV}, E M$ in $10^{52} \mathrm{~cm}^{-3}$ and $L_{\mathrm{X}}$ observed/emitted in $10^{29} \mathrm{erg} \mathrm{s}^{-1}$.

\begin{tabular}{lrrr}
\hline \hline Par. & $08 / 08$ & $17 / 08$ & $06 / 09$ \\
\hline$N_{\mathrm{H}}$ & $1.8_{-0.3}^{+0.2}$ & $1.8_{-0.3}^{+0.2}$ & $1.8_{-0.3}^{+0.2}$ \\
$\mathrm{Fe}$ & $1.07_{-0.20}^{+0.13}$ & $1.07_{-0.20}^{+0.13}$ & $1.07_{-0.20}^{+0.13}$ \\
$\mathrm{Si}$ & $0.64_{-0.27}^{+0.29}$ & $0.64_{-0.27}^{+0.29}$ & $0.64_{-0.27}^{+0.29}$ \\
$\mathrm{O}$ & $0.60_{-0.27}^{+0.19}$ & $0.60_{-0.27}^{+0.19}$ & $0.60_{-0.27}^{+0.19}$ \\
$\mathrm{Ne}$ & $1.38_{-0.31}^{+0.35}$ & $1.38_{-0.31}^{+0.35}$ & $1.38_{-0.31}^{+0.35}$ \\
$k T 1$ & $0.18_{-0.03}^{+0.02}$ & $0.18_{-0.03}^{+0.03}$ & $0.18_{-0.03}^{+0.04}$ \\
$k T 2$ & $0.63_{-0.02}^{+0.02}$ & $0.63_{-0.03}^{+0.03}$ & $0.57_{-0.08}^{+0.03}$ \\
$k T 3$ & $2.97_{-0.18}^{+0.19}$ & $2.50_{-0.40}^{+0.42}$ & $2.31_{-0.51}^{+0.92}$ \\
$E M 1$ & $1.41_{-0.36}^{+0.72}$ & $1.46_{-0.50}^{+0.63}$ & $1.55_{-0.41}^{+0.64}$ \\
$E M 2$ & $2.73_{-0.16}^{+0.18}$ & $2.94_{-0.20}^{+0.27}$ & $1.49_{-0.42}^{+0.13}$ \\
$E M 3$ & $8.72_{-0.32}^{+0.32}$ & $2.94_{-0.33}^{+0.36}$ & $1.37_{-0.18}^{+0.31}$ \\
\hline$\chi_{\text {red }}^{2}$ (d.o.f.) & $1.03(457)$ & $1.02(147)$ & $0.90(112)$ \\
\hline$L_{\mathrm{X}}$ & $13.2(20.9)$ & $6.8(12.5)$ & $3.4(7.1)$ \\
\hline
\end{tabular}

emission since the OM UVW1 is quite sensitive to flux emitted from the accretion spot region.

\subsection{Global spectral properties}

To study the global spectral properties of the X-ray emission from RU Lup and investigate its changes we used the EPIC data. In Fig. 2 we show the PN spectra and the best fit models for the three observations to indicate data quality and spectral changes between the observations. Obviously the spectrum is harder with increasing X-ray brightness and the plasma at hot temperatures is more prominent. Very hot plasma $(z 40 \mathrm{MK})$ is also evidenced by the presence of the $6.7 \mathrm{keV}$ iron line complex in the data from August 8 . To quantify the spectral changes, we fit the EPIC data of the individual observations with a three-temperature model as described above, and present the derived model parameters in Table 2. Note that while absorption and abundances do not vary significantly between the exposures, fits of similar quality can be obtained with a cool component at very low temperatures $(\sim 1 \mathrm{MK})$ and a much larger $E M$, combined with moderately higher values for the oxygen abundance $A_{\mathrm{O}}$ and interstellar column density $N_{\mathrm{H}}$.
We investigated in detail the decline in X-ray brightness throughout the observations and find that there are only marginal changes in the $E M$ of the cool component around $2 \mathrm{MK}$; in contrast, the $E M$ of the hot component decreases significantly by a factor of roughly six. At its brightest phase the EMD is dominated by a hot plasma component at $30 \mathrm{MK}$, and its decline in $E M$ is accompanied by a moderate cooling. In the third observation the intermediate plasma component is also significantly weaker and slightly cooler compared to the other two observations. Consequently, the average coronal temperature drops from $25 \mathrm{MK}$ over $15 \mathrm{MK}$ to $11 \mathrm{MK}$ during the campaign, mainly caused by the decline of EM at temperatures around 20-35 MK. These findings suggest that the fading of RU Lup by a factor of three in emitted $L_{X}$ is caused by a decline of its magnetic activity over a month, and hence that lack of any correlation between X-ray and UV brightness for the three observations is not surprising.

To compare our results with previous X-ray measurements, we also calculated our model fluxes in the Einstein $(0.5-4.0 \mathrm{keV})$ and ROSAT (0.1-2.4 keV) bands. We find that RU Lup was actually in a phase of enhanced activity in autumn 2005, in agreement with the results from our spectral fitting. Even during the third and X-ray darkest XMM-Newton observation, the determined flux of RU Lup is nearly $20 \%$ above the ROSAT value and similar to the Einstein upper limit.

From our spectral fits we derive a moderate absorption column of $N_{\mathrm{H}}=1.8 \times 10^{21} \mathrm{~cm}^{-2}$ when compared to typical values for CTTS (Robrade \& Schmitt 2007). However, recalling that the optical/UV extinction measurements suggest columns of $\sim 10^{20} \mathrm{~cm}^{-2}$ and the fact that RU Lup is viewed close to poleon, the absorption value derived from the X-ray data appears extremely large. To reconcile X-ray and optical/UV absorption columns requires an optically transparent medium with $\mathrm{X}$-ray absorption, an issue we will return to in the next section.

We find no strong overall metal depletion, however, some differences for individual abundances are present. While the modelled abundances of medium FIP $(\mathrm{O}, \mathrm{Si}, \mathrm{S})$ elements are moderately subsolar, the low FIP (Fe, Mg) elements show a higher abundance around or slightly above solar value and especially the high FIP elements (Ne) are enhanced. A high $\mathrm{Ne}$ abundance is commonly observed in CTTS and in active stars, but a high $\mathrm{Fe}$ abundance is rather atypical for a young CTTS. Depending on the specific model we find a Fe/O ratio of 1-1.5 and Ne/O of 2-2.5. Recently, Telleschi et al. (2007) showed that a dependence on spectral type might be present, with G-type CTTS showing a higher Fe abundance (or Fe/O ratio) as CTTS of spectral type K-M. RU Lup's spectral type is usually classified as late $\mathrm{K}$ and its abundance ratios are intermediate between their K-M-type and G-type population, in contrast to their findings. We note that TW Hya, the prototypical accretion dominated CTTS and also of spectral type late $\mathrm{K}$, shows likewise an $\mathrm{Fe} / \mathrm{O}$ ratio of $1-1.5$. Thus spectral type may not be the only relevant parameter for abundance irregularities and accretion properties, for example, may have to be considered.

We also checked for cool plasma which could be responsible for a possible UV/X-ray correlation within individual observations. Since moderate absorption is present in the X-ray spectra, the required plasma temperatures have to be sufficiently high to be responsible for any significant change in the X-ray light curve. Utilising the above derived value of $N_{\mathrm{H}}$, we find that plasma temperatures of roughly $2.5 \mathrm{MK}$ are sufficient, matching the temperature regime where accretion shock plasma is expected. In our 3-T models this corresponds to plasma mainly from the cool and partly from the medium temperature 


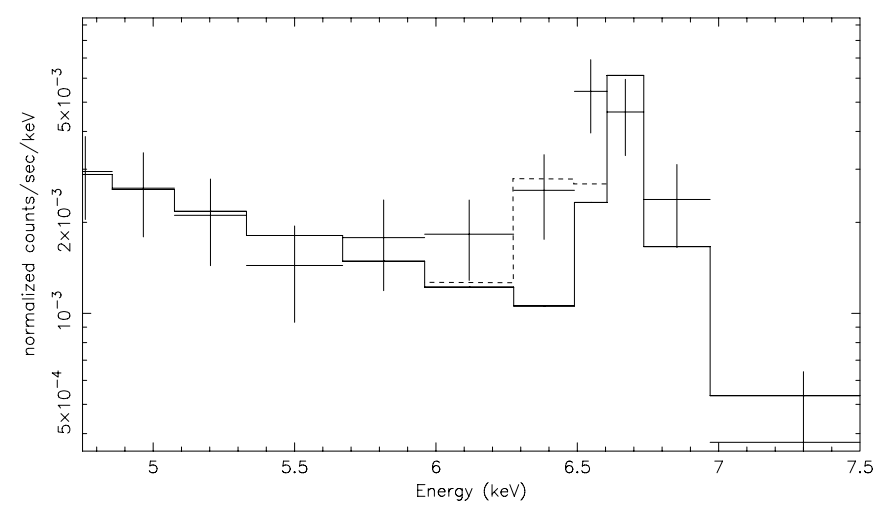

Fig. 3. Spectral region covering the iron $\mathrm{K}_{\alpha}$ line at $6.4 \mathrm{keV}$ and $\mathrm{Fe} \mathrm{XXV}$ line complex at $6.7 \mathrm{keV}$, shown with the three temperature fit (solid) and with additional fluorescence line (dashed).

component. This cooler plasma is obscured by hotter plasma during the more active and X-ray brighter first observation, but becomes dominant especially in the third observation. Therefore the plasma properties derived from the spectral fits are consistent with the presence of a correlation between UV brightness and soft X-ray emission originating from accretion shocks. A stronger accretion component would result in a softening of the $\mathrm{X}$-ray spectra, provided that the usually hotter coronal component is stable. A softening of the X-ray emission with increasing brightness was marginally detected during an XMM-Newton observation of TW Hya (Robrade \& Schmitt 2006), but the count rate of RU Lup is not sufficient to calculate meaningful hardness ratios and investigate this possibility.

\subsection{Fluorescent emission}

In the spectrum of the observation on August 08 some excess emission at energies below the $6.7 \mathrm{keV}$ iron line complex is visible, which might be caused by fluorescence emission from iron $\mathrm{K}_{\alpha}$. After inspection of the PN spectrum in the energy range $4.5-8.0 \mathrm{keV}$ we added a narrow $(10 \mathrm{eV})$ Gaussian component at $6.4 \mathrm{keV}$ to the three temperature model as shown in Fig. 3. We find that the quality of the spectral fit to improves significantly from $\chi_{\text {red }}^{2}=1.33$ (11 d.o.f.) to $\chi_{\text {red }}^{2}=1.03$ (10 d.o.f.). The derived flux from the improved spectral model in the fluorescence line is $5.5 \pm 4.3 \times 10^{-7}$ photons $\mathrm{cm}^{-2} \mathrm{~s}^{-1}$ and the fluorescence line from iron $\mathrm{K}_{\alpha}$ at $6.4 \mathrm{keV}$ is present at high significance $(>90 \%)$.

Under the assumption that the fluorescence emission from iron $\mathrm{K}_{\alpha}$ is produced by the illumination of cooler material with $\mathrm{X}$-ray photons - in principle also electron excitation is possible - the exciting photons need to have energies above $7.11 \mathrm{keV}$ and the illuminated material must be at temperatures of $\lesssim 2 \mathrm{MK}$. While no large flare is present to produce these photons, the hot plasma component might be sufficiently bright to illuminate the star and the disk. Additionally the pole-on geometry of RU Lup is considered optimal for the production of fluorescence emission. The required fluorescence efficiency is of at least $5 \%$, while for the best fit value up to $15-20 \%$ efficiency is necessary. These values are somewhat larger than the typical efficiencies of 3\% derived from Monte Carlo calculations (Bai 1979) to model iron fluorescence emission seen during solar flares. However, on the Sun only the photosphere is illuminated by the X-rays. Larger efficiencies can be obtained from hidden X-ray sources, i.e. active regions on the far side of the star and/or with additional target material. A natural explanation would be the surrounding disk, which is likewise illuminated by the stellar X-ray

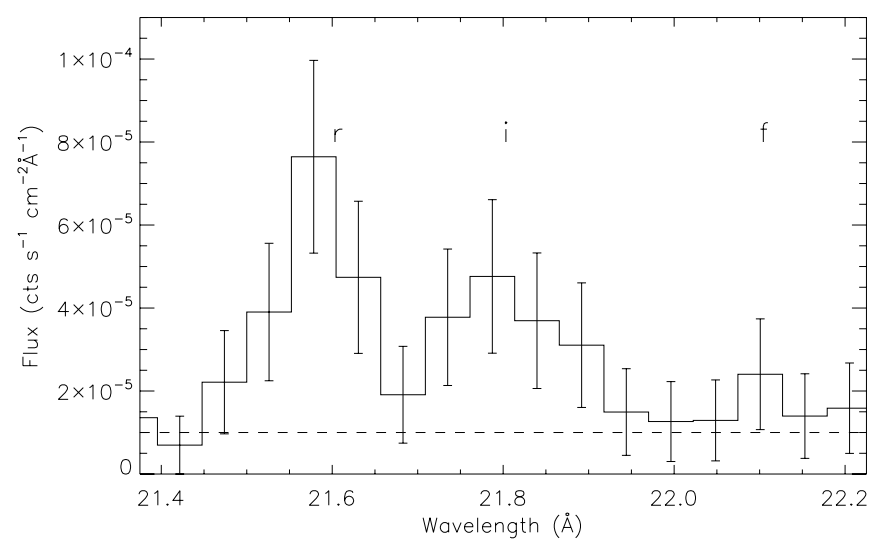

Fig. 4. Fluxed O VII triplet from RGS data (PSF core, rebinned); the background level is indicated by a dashed line.

emission. A strong iron fluorescence line has also been detected in other young stellar objects such as Elias29 (Favata et al. $2005 b$ ) or in the several COUP sources in Orion (Tsujimoto et al. 2005). Theses observations indicate the importance of $\mathrm{X}$-ray emission for the ionisation and consequently temporal evolution of circumstellar disks. While most previous detections are related to strong flaring, $\mathrm{K}_{\alpha}$ emission from Elias29 was also observed in its quiescent state, similar to the detection in RU Lup. Both sources also share the exceptional high coronal iron abundance, which has been interpreted by Favata et al. (2005b) as evidence for X-ray emitting plasma in magnetic flux tubes connecting the star and the circumstellar disk. Magnetic field lines between the star and its disk are a natural ingredient of the magnetospheric accretion model and could provide a flow of fresh disk material into the corona.

\subsection{Oxygen lines - plasma density}

We used high-resolution RGS spectra to measure emission lines from O VII and O VIII, i.e. the resonance $(r)$, intercombination $(i)$ and forbidden $(f)$ lines in the He-like triplet of O VII $(21.6,21.8$, $22.1 \AA$ ) and the Ly $\alpha$ line of O VIII at $18.97 \AA$. We searched for cool excess plasma in CTTS compared to main-sequence stars via the O VIII/O VII(r) line ratio, presented in Sect. 4 and studied the density of the cool (2-3 MK) plasma with the $f / i$-ratio in the He-like triplet of O VII. A density analysis of moderately hotter plasma at $\sim 4 \mathrm{MK}$ could be carried out with the Ne IX triplet, but the unfortunately only moderate SNR of the RGS spectra and non-negligible contamination from the second source (RX J1556.4-3749) in this spectral range prevented this analysis. On the other hand, as shown in Fig. 4, the O VII triplet is clearly detected and shows an intercombination line significantly stronger than the forbidden line. We extracted the line counts from the spectra obtained with the two extraction methods and list them in Table 3. The derived physical properties were then calculated with line intensities corrected for effective area and absorption as adopted from the EPIC model. The plasma density was determined from the relation $f / i=R_{0} /\left(1+\phi / \phi_{\mathrm{c}}+n_{\mathrm{e}} / N_{\mathrm{c}}\right)$ with $f$ and $i$ being the respective line intensities, $R_{0}=3.95$ is the low density limit of the line ratio, $N_{\mathrm{c}}$ the critical density and $\phi / \phi_{\mathrm{c}}$ the radiation term, which is neglected in our calculations as discussed below. The applied values were taken from Pradhan \& Shull (1981).

We find a low $f / i$-ratio independent of the PSF fraction used, While actually the f-line is only marginally detected, formally the derived values are $f / i=0.26 \pm 0.23$ for the PSF core 
Table 3. Measured line counts from RGS data for RU Lup for the two extraction regions described in Sect. 2 .

\begin{tabular}{lrrrrr}
\hline \hline & \multicolumn{2}{c}{ OVIII } & \multicolumn{3}{c}{ OVII - RGS1 } \\
PSF & RGS1 & RGS2 & $r$ & $i$ & $f$ \\
\hline Std. & $28.7 \pm 8.1$ & $33.0 \pm 8.1$ & $22.7 \pm 7.3$ & $20.1 \pm 6.9$ & $8.3 \pm 5.6$ \\
Core & $23.8 \pm 6.4$ & $26.3 \pm 6.2$ & $22.9 \pm 6.1$ & $15.3 \pm 4.0$ & $4.0 \pm 3.2$ \\
\hline
\end{tabular}

and $f / i=0.41 \pm 0.31$ for the standard region. In both cases, the O VII $f / i$-ratio is substantially below unity, resulting in a density of $n_{\mathrm{e}}=4.4(2.7) \times 10^{11} \mathrm{~cm}^{-3}$ for the two extraction methods. The lower limit for the density is $\sim 2(1.5) \times 10^{11} \mathrm{~cm}^{-3}$, on the other hand much higher densities cannot be excluded due to the weakness of the forbidden line. The PSF core result is closer to the theoretically expected g-ratio $(g=(f+i) / r)$ of around unity, but both methods agree within errors. Thus RU Lup is another CTTS that shows a high density in its cool ( $\sim 2 \mathrm{MK})$ plasma component. Two effects, namely the presence of an UV field and of coronal plasma at lower densities, may alter the actual density of the accretion shock plasma in either direction. The strength of the UV field in the proximity of the X-ray emitting plasma is unknown, but its presence may influence the inferred plasma density. A strong UV radiation field would lower the derived plasma densities, however strong UV radiation also has to be attributed to accretion shocks since the photosphere of a late-type star does not produce a sufficiently strong UV flux. On the other hand, a coronal contribution to the cool plasma, which is also present, results in an underprediction of the derived density for the X-ray emitting accretion shock plasma. Therefore we conclude that the bulk of the cool X-ray emitting plasma in RU Lup is generated in accretion shocks.

\section{An X-ray view on accretion and winds in CTTS}

RU Lup is a high accretion rate CTTS and it is useful to compare its properties with those of other X-ray bright CTTS. With the increasing number of available medium and especially highresolution X-ray spectra for these objects we can study signatures of two important processes in CTTS and in star-formation at large, accretion and outflows.

\subsection{Accretion, densities and cool excess}

While the X-ray emission of CTTS at higher energies $(E \gtrsim$ $1 \mathrm{keV}$ ) is dominated by magnetic activity, density analysis with He-like triplets and other line diagnostics suggest that accretion processes contribute significantly to the soft X-ray emission in many, and maybe even all CTTS. Since the disk truncation radii are usually large, the accreting material is infalling with almost free fall velocities and hence post-shock temperatures of up to a few MK are unavoidable. Such plasma is still relatively cool with respect to typical coronal temperatures of active stars and should therefore lead to "cool excess" emission. This opens the possibility to investigate accretion scenarios via cool excess emission by comparing accreting stars with stars where only coronal processes contribute to the X-ray emission.

Instead of using broad band fluxes we use the ratio of two strong lines which are commonly observed in stellar X-ray spectra, the O VIII Ly $\mathrm{Ly}_{\alpha}$ line $(18.97 \AA)$ and the O VII resonance line $(21.6 \AA)$ with peak formation temperatures of $\sim 3 \mathrm{MK}$ and $\sim 2 \mathrm{MK}$ respectively. In Fig. 5 we show the $\mathrm{O}$ VIII $\left(\mathrm{Ly}_{\alpha}\right) / \mathrm{O}$ VII(r) line ratio as calculated with the CHIANTI V 5.2 code

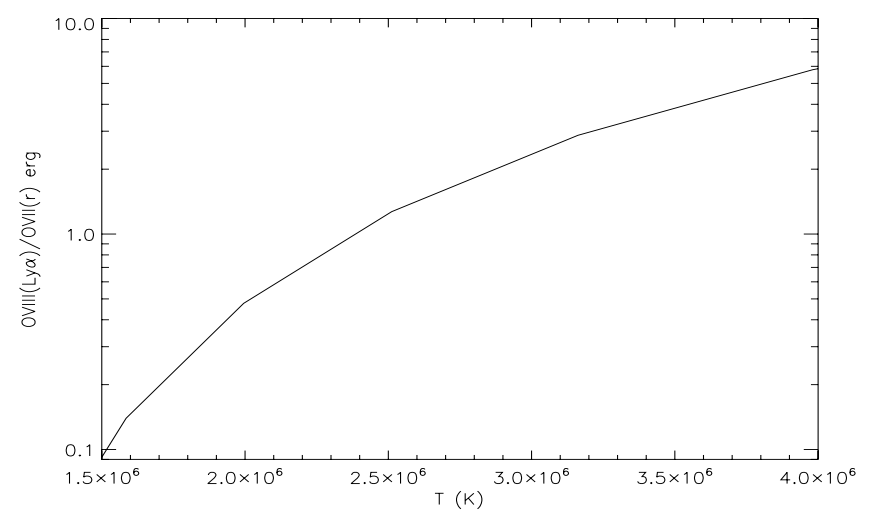

Fig. 5. Theoretical $\mathrm{O}$ VIII $\left(\mathrm{Ly}_{\alpha}\right) / \mathrm{O}$ VII $(r)$ line flux ratio calculated with the Chianti database vs. temperature.

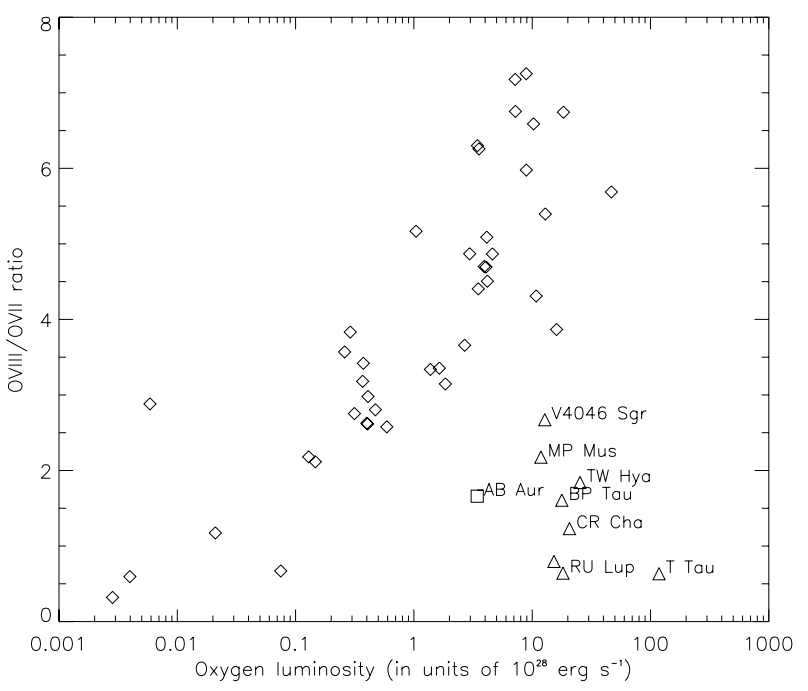

Fig. 6. Ratio of the emitted $\mathrm{O}$ VIII $\left(\mathrm{Ly}_{\alpha}\right) / \mathrm{O}$ VII $(r)$ line flux vs. oxygen luminosity ( $\mathrm{O}$ VII $(r)+\mathrm{O}$ VIII $\left.\left(\mathrm{Ly}_{\alpha}\right)\right)$ for main-sequence stars (diamonds), CTTS (triangles) and the Herbig Ae star AB Aur (square).

(Dere et al. 1997; Landi et al. 2006); it is very sensitive in the temperature range of interest and therefore a powerful diagnostic tool. The emissivity curve of $\mathrm{O}$ VII(r) dominates over the corresponding $\mathrm{O}$ VIII $\left(\mathrm{Ly}_{\alpha}\right)$ curve at temperatures below $\sim 2.5 \mathrm{MK}$, and their ratio is therefore ideal to investigate cool excess plasma as expected from accretion shock models.

Specifically, we compared the ratios of CTTS and other accreting stars with sufficient signal in their high-resolution X-ray spectra to those measured in a large sample of main-sequence stars at various activity levels (Ness et al. 2004). Note that this sample contains several prominent non-accreting young stars such as AB Dor, AT Mic and AU Mic. Results for the Herbig Ae star AB Aur were taken from Telleschi et al. (2007), the O VII $(r)$ line was inferred from their triplet flux assuming a g-ratio of unity, i.e. $(r=f+i)$. For the CTTS sample we adopted the values for BP Tau, CR Cha and TW Hya from Robrade \& Schmitt (2006) with a $g$-ratio of one for CR Cha due to lower SNR, for MP Mus from Argiroffi et al. (2007), for V 4046 Sgr from (Günther et al. 2006) with a distance of $80 \mathrm{pc}$. T Tau is reanalysed here in the same fashion as RU Lup (this work), where both extraction methods are shown. All given values refer to the emitted line fluxes, i.e. they are corrected for the respective absorption column.

In Fig. 6 we plot the absorption corrected O VIII $\left(\mathrm{Ly}_{\alpha}\right) / \mathrm{O} \mathrm{VII}(\mathrm{r})$ line ratio vs. the total oxygen luminosity (the 
power emitted in the $\mathrm{O}$ VIII $\left(\mathrm{Ly}_{\alpha}\right)$ and $\mathrm{O}$ VII $(r)$ lines) for accreting stars and main-sequence stars. The graph clearly shows that our sample CTTS and the main-sequence stars occupy quite different regions and form two very well separated groups in the (O VIII/O VII) vs. (O VIII+O VII) diagram. The correlation between the $\mathrm{O}$ VIII/O VII line ratio and $L_{\text {oxy }}$ for main-sequence stars is well known and caused by the on average higher coronal temperatures in the more active and X-ray brighter stars.

The important point now is that the inferred ratios of all accreting stars lie below the corresponding values for mainsequence stars at the same oxygen luminosity. In other words, at a given oxygen luminosity an accreting star has a smaller $\mathrm{O}$ VIII/O VII line ratio than a non-accreting star. Since this ratio depends only on temperature, there must be additional cool plasma radiating predominantly in the O VII lines in accreting stars, i.e. an cool excess.. We emphasize that the above result is robust; investigating the O VIII and O VII flux alone we find that the respective $\mathrm{O}$ VIII flux is compatible or slightly enhanced, whereas the O VII flux is significantly enhanced in the accreting stars. While most sample CTTS have comparable oxygen luminosities of $1-2 \times 10^{29} \mathrm{erg} \mathrm{s}^{-1}$, corresponding to roughly $5-10 \%$ of their total $L_{\mathrm{X}}$ emitted in these two lines, no correlation between cool excess and oxygen luminosity is present in our sample of accreting stars. Thus the two groups again show different trends, suggesting a different origin of the observed emission.

In the magnetospheric accretion model a larger cool excess corresponds primarily to a higher accretion luminosity. In addition, the precise strength of the cool excess depends on the intrinsic temperature of the accretion shock plasma and the respective coronal contribution to the observed cool plasma, however both are affected by interdependencies and uncertainties that arise from modelling. Besides accretion, other mechanisms have been proposed to produce an excess in the soft X-ray regime, for example disk and coronal stripping of the outer and hottest parts of the corona (Jardine et al. 2006), disruption of hot plasma structures through accreted material (Audard et al. 2005) or filling of coronal structures with cool material (Preibisch et al. 2005). These mechanisms may likewise be present in young stellar objects, however, combining these finding with the often observed high densities in the cool plasma of CTTS, an accretion shock leads to the required additional, persistent, cool, high density plasma component, in a completely natural way.

Note that not all accreting stars generate significant X-ray emission in a high density environment via magnetically funnelled accretion streams. While the exact density of the accretion plasma depends on the presence of UV-fields and the coronal contribution to the cool plasma, all analysed CTTS with a moderate oxygen luminosity exhibit cool plasma at high densities $\left(n_{\mathrm{e}} \gtrsim 10^{11} \mathrm{~cm}^{-3}\right)$. This requires a strong funnelling of the accretion stream, independent of the strength of their cool excess. Contrary, the star with the largest cool excess and especially the largest oxygen luminosity, T Tau itself, is the only T Tauri star that exhibits cool plasma at a lower density and in fact stars like $\mathrm{T}$ Tau are often not classified as CTTS, but as intermediate mass TTS (IMTTS) that are thought to be predecessors or analogs of Herbig AeBe stars. In a related analysis presented by Telleschi et al. (2007), that studies a TTS sample in Taurus-Auriga and shows a soft excess in accreting stars compared to WTTS, the Herbig Ae star AB Aur is found as another example of an accreting star that exhibits a cool excess and a low density cool plasma. Both stars that show no high density plasma are of higher mass $\left(\gtrsim 2 M_{\odot}\right)$ than the sample CTTS $\left(\lesssim 1 M_{\odot}\right)$, suggesting a scenario where accretion properties are linked to stellar properties, i.e. mass and radius.
Table 4. Properties of accreting stars and their cool plasma. Masses and radii from the references in this section and therein, $M^{-1 / 2} R^{-3 / 2}$ in solar units, $\mathrm{TS}=3.44 \times M / R$ (shock temp.), Cor.C. $=$ coronal contribution.

\begin{tabular}{|c|c|c|c|c|c|c|c|}
\hline Star & $\begin{array}{c}M \\
M_{\odot}\end{array}$ & $\begin{array}{r}R \\
R_{\odot}\end{array}$ & $M^{-1 / 2} R^{-3 / 2}$ & $\begin{array}{c}\text { Density } \\
10^{11} \mathrm{~cm}^{-3}\end{array}$ & $\begin{array}{c}\mathrm{TS} \\
\mathrm{MK}\end{array}$ & $\begin{array}{c}\text { Cor.C. } \\
(E M 1 / E M 2)\end{array}$ & Cool Ex. \\
\hline TW Hya & 0.7 & 1.0 & 1.20 & vhigh 21. & 2.4 & weak(16.) & mod. \\
\hline RU Lup & 0.8 & 1.7 & 0.50 & hig & 1.6 & mo & strong \\
\hline BP Tau & 0.8 & 2.0 & 0.40 & high 3.2 & 1.4 & mo & mod. \\
\hline MP Mus & 1.2 & 1.3 & 0.62 & high 5.0 & 3.2 & mod. & weak \\
\hline T Tau & 2.4 & 3.6 & 0.09 & low 0.1 & 2.3 & weak(2.1) & strong \\
\hline AB Aur & 2.7 & 2.3 & 0.17 & low 0.1 & 4.0 & mod.(0.6) & weak \\
\hline
\end{tabular}

\subsection{Accretion models vs. observations}

We then compared our results with expected accretion shock parameters from calculations based on the magnetically funnelled accretion model described in Calvet \& Gullbring (1998). Discarding two object from the discussion, V 4046 Sgr (K5+K7, age $\sim 6 \mathrm{Myr}$, Quast et al. 2000) since it is a binary and the distribution of its X-ray emission among the binary components is unknown and CR Cha (K2, age $\sim 1 \mathrm{Myr}$ ) since its stellar parameters are only poorly constrained, we summarise the stellar properties of the accreting stars and their fitting results in Table 4. Filling factors as derived by Calvet \& Gullbring (1998) are in the range of $f=0.01$ for magnetically funneled accretion on CTTS, e.g. $f=0.007$ for BP Tau, but can be up to an order of magnitude smaller or larger in individual stars. Adopting their formulae and assuming free-fall velocity, i.e. the disk truncation radius to be much larger than the stellar radius, the infall (postshock $\left.n_{\mathrm{p}}=4 \times n_{\mathrm{i}}\right)$ density is $n_{\mathrm{i}} \sim \dot{M} \times f^{-1} \times\left(M^{-1 / 2} R^{-3 / 2}\right)$. The accretion luminosity is $L_{\mathrm{Acc}} \sim \dot{M} \times M / R$, the corresponding plasma temperature is $T \sim V^{2} \sim M / R$ and therefore assumed to be independent of funnelling. Note that different shock temperatures also influence the observed $\mathrm{O}$ VIII/O VII ratio and some uncertainties on the stellar parameters are also present for the investigated stars. The coronal contribution to the X-ray emission from the cool plasma is approximated by the ratio $E M 1 / E M 2$ in the respective $3-\mathrm{T}$ models, in our sample stars the corresponding temperatures are 2-3 MK (T1) and 6-7 MK (T2). Assuming similar shaped underlying stellar coronal EMDs as determined from active stars, this ratio traces the importance of the coronal contribution to the cool plasma. We denote $E M 1 / E M 2$ ratios above 2 as "weak", in the range $0.5-2$ as "moderate" and below 0.5 as "strong". Finally we roughly quantified the strength of the cool excess by comparing the $\mathrm{O}$ VIII $\left(\mathrm{Ly}_{\alpha}\right) / \mathrm{O} \mathrm{VII}(r)$ line ratio for the coronal sources that exhibit the lowest value at a given oxygen luminosity and the respective accreting sources. We denote a cool excess as "weak" for a ratio $\lesssim 2$, "moderate" for a ratio of $\sim 2-4$ and "strong" for larger ratios.

Altogether the characteristics of the accreted plasma depend on an interplay of stellar properties and the accretion stream. The dependence on stellar properties clearly favours the high density scenario for more compact low-mass stars, and predicts lower plasma temperatures and therefore larger soft excess for less contracted objects as long as X-ray temperatures are reached in the accretion shock. Considering the accretion stream, the ratio $\dot{M} / f$ is important for the resulting density, i.e. large mass accretion rates and small filling factors are favoured in the higher density regime. A strong cool excess is produced by large mass accretion rates via a large accretion luminosity. The accretion luminosity also increases for more compact objects, but when 
considering a cool excess as measured by O VIII/O VII it conflicts with the corresponding higher shock temperatures.

There is a remarkable correlation between the cool plasma properties as expected in the magnetospheric accretion model from stellar parameters alone and the observed properties for the six investigated stars. Notably the measured densities correlate well with the value of the quantity $M^{-1 / 2} R^{-3 / 2}$ suggesting that the ratio $\dot{M} / f$ does not vary extremely between the sample CTTS and that the cool plasma is indeed dominated by the accretion process in most cases. The property $M^{-1 / 2} R^{-3 / 2}$ describes on the one hand the compactness of a star for a given mass but also some kind of general smallness. The only star with very high density $\left(n_{\mathrm{e}}>10^{12} \mathrm{~cm}^{-2}\right)$, TW Hya, is also the only star with $\left(M^{-1 / 2} R^{-3 / 2}\right)>1$, all stars with $0.4 \lesssim\left(M^{-1 / 2} R^{-3 / 2}\right) \lesssim 0.6$ show high densities $\left(n_{\mathrm{e}}>10^{11} \mathrm{~cm}^{-2}\right)$ and the other two stars with low densities $\left(n_{e} \leq 10^{10} \mathrm{~cm}^{-2}\right)$ have $\left(M^{-1 / 2} R^{-3 / 2}\right)<0.2$. While among the high density stars the correlation is roughly linear, the larger variations in density compared to those of $M^{-1 / 2} R^{-3 / 2}$ for the extreme cases of very high and low density suggest that an additional correlation with $\dot{M} / f$ might amplify this trend. Consequently the funnelling would have to be very effective for compact and tiny objects and much less pronounced for expanded or more massive stars. Note, that the density of the cool plasma component does not correlate with either the shock temperature or with the cool excess of the respective star.

The temperature of the accreted plasma is also determined by stellar mass and radius, but for the strength of the cool excess the coronal contribution and its X-ray brightness relative to the one of the accretion spot is more important, since our definition of a cool excess involves O VIII emission which traces hotter plasma. Necessarily the intrinsic temperature of the shocked plasma, which depends on the ratio $\mathrm{M} / \mathrm{R}$, has to be sufficiently low to produce a cool excess that is traced by enhanced O VII emission. The calculated shock temperatures are in the range between $1.5-4.0 \mathrm{MK}$, i.e. the temperature range where the ionisation stage changes from a dominating O VII contribution to a dominating O VIII contribution in a collisionally ionised plasma. In two stars, MP Mus and AB Aur, the calculated shock temperature is already above $3 \mathrm{MK}$ and their cool excess is indeed weakest in our sample. That the Herbig AeBe star AB Aur shows some cool excess at all is surprising given its shock temperature of $4 \mathrm{MK}$. This might be explained by inhomogeneous accretion spots that arise from recent modelling (Romanova et al. 2004). These spots show for example an intrinsic temperature distribution, an effect that might be more pronounced especially for less funneled accretion streams. A strong cool excess is only observed in stars with shock temperatures below $2.5 \mathrm{MK}$, but stars with similar shock temperatures may show quite different other accretion properties as discussed exemplarily for the extreme cases T Tau and TW Hya. In T Tau a large mass accretion rate of 3-6 $\times 10^{-8} M_{\odot} / \mathrm{yr}$ (Calvet et al. 2004), moderate shock temperatures and a weak coronal contribution are present, producing the largest cool excess in our sample; its lower plasma density requires a large filling factor and/or possibly involves other "cooling mechanisms" like mass loading of coronal structures as suggested by Güdel et al. (2007). On the other hand, TW Hya, the exceptional case of an accretion dominated CTTS shows no extreme cool excess. However, its shock temperature is only average and in addition the lower mass accretion rate of $\sim 10^{-9} M_{\odot} /$ yr (Muzerolle et al. 2000; Alencar \& Batalha 2002) contributes to TW Hya's only moderate cool excess. Its high plasma density then points to a strong funnelling of the accreted plasma. Further modifications, for example through different disk truncation radii or levels of stellar activity, seem to have only a minor impact on the cool excess at least in CTTS. We note that CR Cha and V 4046 Sgr mostly fit into this picture since they are K-type low-mass stars and also show the expected high plasma density. Admittedly our sample is not very large and completely lacking of any CTTS with very low mass $\left(\lesssim 0.5 M_{\odot}\right)$ due to the fact that they are usually X-ray darker since $\mathrm{L}_{\mathrm{X}}$ correlates with mass in T Tauri stars. If also applicable in this mass regime, we expect early M-type CTTS to show rather high plasma densities that should be observable in X-rays, if accretion rates are high enough and the accretion plasma is heated to sufficiently high temperatures.

One parameter, i.e. stellar age, is completely absent in the above discussion. The stellar evolution is of course "hidden" in the stellar radius for a given mass and consequently old ( $110 \mathrm{My}$ ) and more compact CTTS like TW Hya or MP Mus exhibit higher shock temperatures than young ( $\lesssim 1 \mathrm{My)}$ CTTS such as BP Tau. Likewise the mass accretion rates of CTTS decrease on average throughout their evolution, further diminishing the cool excess with time. While there is probably a large spread, this trend is also present in the observations and none of the older CTTS, TW Hya, MP Mus and V 4046 Sgr, exhibit any strong cool excess. On the other hand, all older CTTS show quite high plasma densities suggesting moderate mass accretion rates and a well funneled accretion stream. This proposal of a unified scenario for the observed X-ray properties of accreting stars needs to be tested with a larger sample to improve the statistics especially among the very low and intermediate mass regime, but provides a plausible explanation for the similarities as well as the differences in the observed characteristics of X-ray bright accreting stars.

\subsection{Outflows and anomalous $X$-ray absorption}

In the X-ray spectra of the almost pole-on RU Lup we measure an absorbing column density clearly incompatible with optical or UV measurements. In Fig. 7 we show the X-ray spectrum of RU Lup as observed on August 08 with our best fit model and also with the best fit model using the absorption value derived from UV measurements Herczeg et al. (2005). Column densities derived from optical observations are usually comparable or even lower. Besides the fact that the applied model is quite unphysical since it does not contain any cool plasma, it still cannot explain the observed spectrum. Therefore we have to attribute the observed discrepancy to the presence of an unknown X-ray absorbing material along the line of sight. The same effect was also detected in several other CTTS such as AA Tau (Schmitt \& Robrade 2007), a CTTS viewed under an intermediate inclination of $\sim 75^{\circ}$ (Bouvier et al. 1999) and in the also pole-on CTTS TW Hya (Robrade \& Schmitt 2006), where the effect is an order of magnitude lower and the discrepancy could be instead attributed to modelling or calibration uncertainties.

Reconciliation of the X-ray and UV/optical absorption observations requires an optically transparent, but sufficiently X-ray absorbing medium. This medium needs to be free of any significant contribution of larger dust grains but has to contain a lot of X-ray absorbing material such as gas. Additionally the absorption component has to be persistent and relatively steady over at least a month. A good candidate for such a medium could be a strong outflow either from the star, possibly powered by the accretion process (Matt \& Pudritz 2005), and/or from the inner disk (Konigl \& Pudritz 2000; Alencar et al. 2005). Likewise the accretion streams may contribute to the additional absorption. Another more exotic possibility could be a dust envelope with a very peculiar dust grain distribution containing sufficiently small 


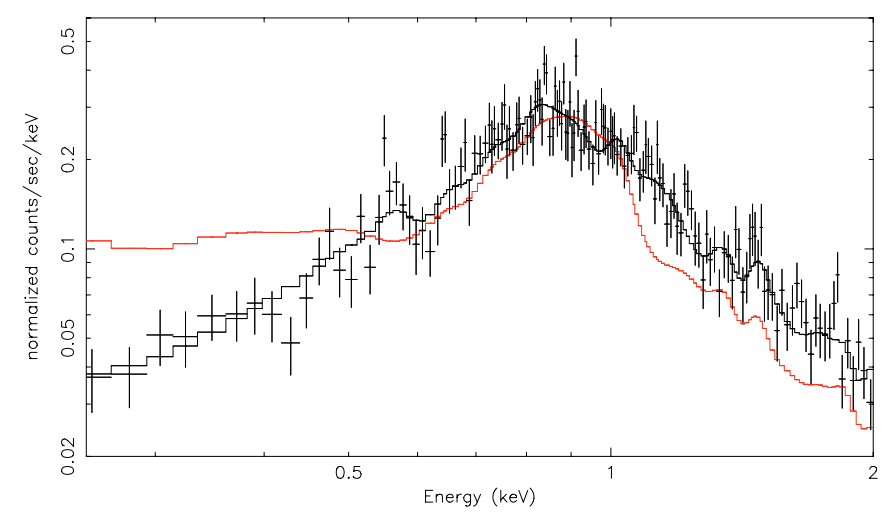

Fig. 7. X-ray spectra of the almost pole-on CTTS RU Lup. The histograms show our model (black) and the best fit model with absorption set to values derived from the UV measurements (red).

grains. Considering the nearly pole-on view of RU Lup, a wind scenario appears natural, in particular, since strong, fast winds are well known for CTTS. However, the origin and geometry of the wind strongly depend on the underlying model and detailed properties of CTTS winds are the subject of considerable debate.

In general, a stellar wind requires lower mass outflow rates than a disk wind, since it originates from the stellar surface and therefore obscures the X-ray emitting regions more effectively. On the other hand, a stellar wind originating from coronal structures in analogy to the solar wind would have temperatures of at least several $100000 \mathrm{~K}$, much higher than the few $10000 \mathrm{~K}$ usually attributed to CTTS winds. A hot wind has been claimed on the basis of asymmetric O VI line profiles observed in TW Hya by Dupree et al. (2005); however, Johns-Krull \& Herczeg (2007) question this interpretation and argue for a much cooler wind in TW Hya. Unfortunately our data do not allow us to investigate the properties of the absorbing wind component in more detail.

If we adopt - as the simplest absorption model - that of a spherically symmetric wind with constant velocity, one derives a line-of-sight absorption column of $N_{\mathrm{H}}=n_{0} \times R_{0}$ with $n_{0}$ denoting the plasma density at the wind base and $R_{0}$ its distance from the star. Assuming the wind base to be essentially at the surface of the star and adopting the numbers of RU Lup, i.e. $N_{\mathrm{H}} \sim 1.7 \times 10^{21} \mathrm{~cm}^{-2}$ and $R_{0}=1.7 R_{\odot}$, we find a base density of $1.4 \times 10^{10} \mathrm{~cm}^{-3}$. Further assuming this outflow to occur over $4 \pi$ steradian with a velocity of $300 \mathrm{~km} \mathrm{~s}^{-1}$, results in a mass loss rate of $\dot{M}_{\text {wind }} \sim 2 \times 10^{-9} M_{\odot} /$ yr. However, the otherwise derived mass loss rates of CTTS are extremely uncertain, but are thought to be in the range $0.01-0.1$ of the respective mass accretion rates (Hartigan et al. 1995; Konigl \& Pudritz 2000), which in turn are also only poorly constrained and in addition time variable. Recent estimates for the mass accretion rates for RU Lup are about $5 \pm 2 \times 10^{-8} M_{\odot} / \mathrm{yr}$ (Herczeg et al. 2005), and therefore a mass loss rate of $\dot{M}_{\text {wind }} \sim 2 \times 10^{-9} M_{\odot} /$ yr would perfectly fit the "expectations". Carrying out the same exercise for TW Hya $\left(N_{\mathrm{H}} \sim 3 \times 10^{20} \mathrm{~cm}^{-2}\right)$ yields again consistent results if one adopts mass accretion rates of $0.5-2 \times 10^{-9} M_{\odot} / \mathrm{yr}$ (Muzerolle et al. 2000; Alencar \& Batalha 2002). Note that outflow velocity, wind solid angle and location of the wind base appear in this model only as linear parameters and moderate changes of their values do not change the rough estimations of the mass loss rates given above. Accordingly, the lower X-ray absorption for TW Hya would correspond to a more moderate wind, for RU Lup both values are roughly by a magnitude larger and may well be explained in a similar, scaled up scenario. AA Tau is viewed over the rim of a warped disk which partially occults the star at recurring phases (Bouvier et al. 1999) and associated material, accretion streams or outflows from the disk likely add significantly to its large and variable X-ray absorption (Schmitt \& Robrade 2007).

We therefore conclude that the available X-ray data can indeed constrain the amount of material along the line of sight and yield - when adopting a simplified wind model with an outflow velocity of a few hundred $\mathrm{km} \mathrm{s}^{-1}-$ mass loss rates at the percent level of the mass accretion rates. Interpreting therefore the observed X-ray absorption as arising from a wind thus yields at least a physically consistent picture.

\section{Conclusions}

From our studies of the X-ray emission from RU Lup and other CTTS we draw the following conclusions:

1. RU Lup is another example of a CTTS where cool high density plasma is present. The density of $3-4 \times 10^{11} \mathrm{~cm}^{-3}$ as deduced from the $\mathrm{O}$ VII triplet supports an accretion shock scenario for the bulk of cool plasma. This cool plasma component is quite stable over a month.

2. Spectral variations indicate a change from an EMD that is clearly dominated by coronal activity in the first observation to a phase with a more equal distribution of cooler and hotter plasma in observation three. Many examples of coronal dominated CTTS have been detected so far, but RU Lup is a rare example where a change from state dominated by coronal activity to a state with a major contributing cool accretion component is observed over a month. Its X-ray luminosity is not correlated with UV brightness over the campaign, however during X-ray fainter phases there are indications for a correlation on timescales of several hours.

3. In all investigated accreting stars the characteristics of the cooler X-ray emitting plasma are influenced by the accretion process. An excess of cool plasma, as evidenced by a lower $\mathrm{O}$ VIII $\left(\mathrm{Ly}_{\alpha}\right) / \mathrm{O}$ VII $(r)$ line-ratio, is present in our sample stars when compared to main-sequence stars. The strength of the cool excess depends on an interplay of accretion shock luminosity and temperature as well as the omnipresent coronal contribution. Cool, high density plasma is found so far exclusively in the low-mass CTTS sample $\lesssim 1 M_{\odot}$ ), while accreting stars with intermediate mass $\left(\gtrsim 2 M_{\odot}\right)$ show lower densities. Many aspects of the accretion process can be explained by stellar mass and radius and their evolution with time in a qualitative way. We suspect a relation to mass accretion rates and especially amount of funnelling, which produce the different properties of the accretion shock plasma that are seen in the respective X-ray spectra.

4. We derive from X-ray spectra an absorption column of $1.8 \times 10^{21} \mathrm{~cm}^{-2}$ for RU Lup, roughly an order of magnitude above the value derive from $\mathrm{Ly}_{\alpha}$ absorption. To reconcile the optical extinction and X-ray absorption towards RU Lup, one needs X-ray absorption in an optically almost transparent medium. Large discrepancies between absorption values derived from X-ray spectra and in the optical/UV wavelength regimes are also found in several other CTTS. We suggest that strong outflows/winds are responsible, which originate either from the star and/or the disk. Clearly strong winds are present in RU Lup and the nearly pole-on view does not favour anomalous dust material to be responsible for the excess absorption; however, a contribution from the accretion 
flows is expected. Using the required additional X-ray absorption columns and a simple wind model we derive mass loss rates of a few percent of the stellar mass accretion rates for CTTS.

Acknowledgements. This work is based on observations obtained with $X M M-N e w t o n$, an ESA science mission with instruments and contributions directly funded by ESA Member States and the USA (NASA). J.R. acknowledges support from the DLR under grant 50OR0105.

\section{References}

Alencar, S. H. P., Basri, G., Hartmann, L., \& Calvet, N. 2005, A\&A, 440, 595 Alencar, S. H. P., \& Batalha, C. 2002, ApJ, 571, 378

Appenzeller, I., Krautter, J., \& Jankovics, I. 1983, A\&AS, 53, 291

Argiroffi, C., Maggio, A., \& Peres, G. 2007, A\&A, 465, L5

Arnaud, K. A. 1996, in Astronomical Data Analysis Software and Systems V, ed. G. H. Jacoby, \& J. Barnes, ASP Conf. Ser., 101, 17

Audard, M., Güdel, M., Skinner, S. L., et al. 2005, ApJ, 635, L81

Bai, T. 1979, Sol. Phys., 62, 113

Bouvier, J., Chelli, A., Allain, S., et al. 1999, A\&A, 349, 619

Calvet, N., \& Gullbring, E. 1998, ApJ, 509, 802

Calvet, N., Muzerolle, J., Briceño, C., et al. 2004, AJ, 128, 1294

Dere, K. P., Landi, E., Mason, H. E., Monsignori Fossi, B. C., \& Young, P. R. 1997, A\&AS, 125, 149

Dupree, A. K., Brickhouse, N. S., Smith, G. H., \& Strader, J. 2005, ApJ, 625, L131

Favata, F., Flaccomio, E., Reale, F., et al. 2005a, ApJS, 160, 469

Favata, F., Micela, G., Silva, B., Sciortino, S., \& Tsujimoto, M. 2005b, A\&A, 433, 1047

Favata, F., Bonito, R., Micela, G., et al. 2006, A\&A, 450, L17

Feigelson, E. D., \& Montmerle, T. 1999, ARA\&A, 37, 363

Gahm, G. F. 1980, ApJ, 242, L163

Grevesse, N., \& Sauval, A. J. 1998, Space Sci. Rev., 85, 161

Güdel, M., Skinner, S. L., Briggs, K. R., et al. 2005, ApJ, 626, L53

Güdel, M., Skinner, S. L., Mel'Nikov, S. Y., et al. 2007, A\&A, 468, 529

Günther, H. M., Liefke, C., Schmitt, J. H. M. M., Robrade, J., \& Ness, J.-U. 2006, A\&A, 459, L29

Günther, H. M., Schmitt, J. H. M. M., Robrade, J., \& Liefke, C. 2007, A\&A, 466, 1111
Hartigan, P., Edwards, S., \& Ghandour, L. 1995, ApJ, 452, 736

Herczeg, G. J., Walter, F. M., Linsky, J. L., et al. 2005, AJ, 129, 2777

Hughes, J., Hartigan, P., \& Clampitt, L. 1993, AJ, 105, 571

Jardine, M., Cameron, A. C., Donati, J.-F., Gregory, S. G., \& Wood, K. 2006, MNRAS, 367, 917

Johns-Krull, C. M., \& Herczeg, G. J. 2007, ApJ, 655, 345

Kashyap, V., \& Drake, J. J. 2000, Bull. Astron. Soc. Ind., 28, 475

Kastner, J. H., Huenemoerder, D. P., Schulz, N. S., Canizares, C. R., \& Weintraub, D. A. 2002, ApJ, 567, 434

Konigl, A., \& Pudritz, R. E. 2000, Protostars and Planets IV, 759

Lamzin, S. A. 2000, Astron. Lett., 26, 225

Lamzin, S. A., Bisnovatyi-Kogan, G. S., Errico, L., et al. 1996, A\&A, 306, 877

Landi, E., Del Zanna, G., Young, P. R., et al. 2006, ApJS, 162, 261

Loiseau, N., Ehle, M., Pollock, A., et al. 2006

Matt, S., \& Pudritz, R. E. 2005, ApJ, 632, L135

Muzerolle, J., Calvet, N., Briceño, C., Hartmann, L., \& Hillenbrand, L. 2000, ApJ, 535, L47

Ness, J.-U., \& Wichmann, R. 2002, Astron. Nachr., 323, 129

Ness, J.-U., Güdel, M., Schmitt, J. H. M. M., Audard, M., \& Telleschi, A. 2004, A\&A, 427, 667

Neuhäuser, R., Sterzik, M. F., Schmitt, J. H. M. M., Wichmann, R., \& Krautter, J. 1995, A\&A, 297, 391

Pradhan, A. K., \& Shull, J. M. 1981, ApJ, 249, 821

Pravdo, S. H., Feigelson, E. D., Garmire, G., et al. 2001, Nature, 413, 708

Preibisch, T., Kim, Y.-C., Favata, F., et al. 2005, ApJS, 160, 401

Quast, G. R., Torres, C. A. O., de La Reza, R., da Silva, L., \& Mayor, M. 2000, in IAU Symposium

Robrade, J., \& Schmitt, J. H. M. M. 2006, A\&A, 449, 737

Robrade, J., \& Schmitt, J. H. M. M. 2007, A\&A, 461, 669

Romanova, M. M., Ustyugova, G. V., Koldoba, A. V., \& Lovelace, R. V. E. 2004, ApJ, 610, 920

Schmitt, J. H. M. M., \& Liefke, C. 2004, A\&A, 417, 651

Schmitt, J. H. M. M., \& Robrade, J. 2007, A\&A, 462, L41

Schmitt, J. H. M. M., Robrade, J., Ness, J.-U., Favata, F., \& Stelzer, B. 2005, A\&A, 432, L35

Smith, R. K., Brickhouse, N. S., Liedahl, D. A., \& Raymond, J. C. 2001, ApJ, 556, L91

Stelzer, B., \& Schmitt, J. H. M. M. 2004, A\&A, 418, 687

Stempels, H. C., \& Piskunov, N. 2002, A\&A, 391, 595

Telleschi, A., Güdel, M., Briggs, K. R., Audard, M., \& Scelsi, L. 2007, A\&A, 468,443

Tsujimoto, M., Feigelson, E. D., Grosso, N., et al. 2005, ApJS, 160, 503 